\title{
SELEÇÃO DE ISOLADOS DE BACILLUS THURINGIENSIS BERLINER PARA TETRANYCHUS URTICAE KOCH
}

\section{L.F.V. Silveira, R.A. Polanczyk, D. Pratissoli, C.R. Franco}

Universidade Federal do Espírito Santo, Centro de Ciências Agrárias, Departamento de Produção Vegetal, NUDEMAFI, Laboratório de Entomologia, CP 16, CEP 29500-000, Alegre, ES, Brasil. E-mail: viannasilveira@ gmail.com

\section{RESUMO}

\begin{abstract}
O ácaro rajado é uma das pragas mais importantes em culturas no Brasil. O uso do controle químico de forma descontrolada leva a sérias consequências, como a presença de resíduos em frutos e surgimento de populações resistentes. Faz-se necessário o uso de alternativas de manejo do ácaro, como o controle biológico, que vem sendo utilizado como ferramenta no manejo de diversas pragas de importância agrícola no mundo. Para a realização dos bioensaios, foram avaliadas 120 cepas de Bacillus thuringiensis Berliner quanto à patogenicidade para Tetranychus urticae Koch. Foram preparadas suspensões das diferentes cepas contendo $3 \times 10^{8}$ esporos viáveis $/ \mathrm{mL}$ de água destilada, aplicadas em discos de folhas de feijão de porco (Canavalia ensiformes DC). Cada cepa constituiu-se em um tratamento, com 8 repetições, cada uma contendo 10 fêmeas adultas do ácaro. Os testes em condições de laboratório mostraram que a mortalidade foi significativa, variando de 6,7\% (cepa LMJ7A) a 54,9\% (cepas 1077B e 886F).
\end{abstract}

PALAVRAS-CHAVE: Controle biológico, entomopatógeno, bactéria, toxinas cry, ácaro.

\begin{abstract}
SCREENINGOF BACILLUSTHURINGIENSISBERLINERAGAINST TETRANYCHUSURTICAE $\mathrm{KOCH}$. The two-spotted spider mite is the most important pest to Brazilian crops. The incorrect use of chemical control leads to serious consequences, such as the presence of residues in fruits and the emergence of resistant populations. There is a need for alternative methods for managing the mite, such as biological control, which has been used as a tool in the management of various pests of agricultural importance in the world. Bioassays were carried out to analyze 120 strains of Bacillus thuringiensis Berliner in regard to their pathogenicity to Tetranychus urticae Koch. Suspensions were prepared of the different strains, containing $3 \times 10^{8}$ spores $/ \mathrm{mL}$ of distilled water, and applied on Jack-bean (Canavalia ensiformes DC) leaf disks. Each strain constituted a treatment, with 8 repetitions, each containing 10 adult Tetranychus urticae females. The tests in laboratory conditions showed that mortality was significantly, ranging from 6.7\% (strains LMJ7A) to 54.9\% (strains 1077B and 886f).
\end{abstract}

KEY WORDS: Biological control, entomopathogen, bacterial, cry toxins, mite.

\section{INTRODUÇÃO}

O ácaro rajado Tetranychus urticae Koch (Tetranychidae) é uma das pragas mais importantes na agricultura, atacando várias espécies de plantas em várias regiões do país, considerado uma espécie cosmopolita e polífaga (Moraes; FLECHTMANN, 2008).

Atualmente, o controle de T. urticae é realizado por meio de acaricidas químicos, utilizados indiscriminadamente, podendolevar a vários problemas, como contaminação do meio ambiente, eliminação de inimigos naturais, intoxicações, tanto de produtores como consumidores e surgimento de populações resistentes, sendo que este último problema já vem preocupando a comunidadecientífica desde a década de 70 (Silva, 1971; Watanabe et al., 1994; Poletti et al., 2006; SATO et al., 2007).

Comisso, novas ferramentas de manejo de pragas devem ser disponibilizadas aos produtores, como o controle biológico com predadores e patógenos (PolanczyK et al., 2004; Collier et al., 2007; Fatoretto et al., 2007), assim como de sua conservação, utilizando agrotóxicos seletivos (MONTEIRO, 2001; POLETTI et al., 2008).

Dentro do grupo dos patógenos, as bactérias pertencentes à família Bacillaceae, principalmente a espécie Bacillus thuringiensis, têm comprovada 
atividade tóxica em diversas famílias de insetos e ácaros, e com grande vantagem por apresentar inocuidade aos mamíferos e vertebrados e ausência de toxicidade às plantas (WHiteley; SCHNEPF, 1986; Glare; O'Callagham, 2000; Hansen; Salamitou, 2000).

$\mathrm{O}$ estudo com fungos entomopatogênicos para o controle de ácaros é mais difundido e apresenta excelentes resultados (TAMAI et al., 1999; TAMAI et al., 2002; OliveIRA et al., 2004). Assim, visando maior variabilidade de princípios ativos, e uma alternativa ao manejo da praga em questão, o objetivo desse trabalho é selecionar cepas de $B$. thuringiensis com potencial para o manejo do ácaro rajado T. urticae.

\section{MATERIAL E MÉTODOS}

Criação do ácaro rajado. A criação estoque iniciouse a partir de adultos de T. urticae coletados em campo sob folhas de mamão Carica papaya L., no Município de Linhares (ES), em maio de 2006. No laboratório, foram transferidos para folhas de feijão-de-porco Canavalia ensiformis DC. mantidos em pratos plásticos $(20 \mathrm{~cm}$ de diâmetro), sobre manta acrílica umedecida com água destilada e coberta com papel filtro. Os pratos contendo os ácaros foram mantidos em câmaras climatizadas reguladas à temperatura $26 \pm 1^{\circ} \mathrm{C}, 70 \pm 10 \%$ UR e fotofase de $12 \mathrm{~h}$, sendo as folhas renovadas a cada 5 a 7 dias.

Testes de patogenicidade. Foram utilizadas 120 cepas de B. thuringiensis cedidas pelo Laboratório de Genética de Bactérias e Biotecnologia Aplicada (LGBBA), junto ao Departamento de Biologia Aplicada a Agropecuária na Faculdade de Ciências Agrárias e Veterinárias, Unesp, Campus de Jaboticabal, SP. As cepas desta coleção, coletadas em solo e provenientes de diferentes locais, foram estocadas em fitas de papel filtro impregnados com uma suspensão de esporos, e mantidos em tubos tipo eppendorf a $5^{\circ} \mathrm{C}$.

Estes isolados foram multiplicados em meio de cultura BHI (infusão de cérebro e coração) a $30^{\circ} \mathrm{C}$, sob agitação orbital a $180 \mathrm{rpm}$ por 48 horas. Após a lise bacteriana, a mistura contendo esporos, cristais e células vegetativas foi submetida a três centrifugações consecutivas de $5.000 \mathrm{rpm}$, por 20 minutos eliminando-se o sobrenadante. Então, uma alíquota de $1 \mathrm{~mL}$ foi diluída 1.000 vezes em água destilada autoclavada, e a concentração de esporos foi determinada conforme método descrito em Alves; MORAES (1998). Em seguida, foram preparadas suspensões de $B$. thuringiensis, contendo $3 \times 10^{8}$ esporos viáveis/ $\mathrm{mL}$ de água destilada autoclavada.

Em cada experimento foram utilizadas 30 cepas, totalizandoquatrogruposcom120cepasnototal(Tabela1).

Discos de folhas de feijão-de-porco de $4,5 \mathrm{~cm}$ de diâmetro foram imersos durante 5 segundos nas suspensões previamente preparadas. A testemunha foi imersa em água destilada.
Após a imersão nas suspensões, os discos foram colocados para secar a temperatura ambiente por 15 minutos e depois foram acondicionados sobre uma pequena placa de isopor flutuando na água, fixado no centro de uma placa de gerbox (diâmetro de $6 \mathrm{~cm}$ ) por umalfinetecoladocom colaabasedesilicone, deixandose um disco por placa, sendo esta unidade considerada uma repetição, tendo sido preparadas 8 repetições.

Após esta etapa, foram transferidas 10 fêmeas adultas de T. urticae por disco e as placas foram mantidas em câmara climatizada a temperatura de $26 \pm$ $1^{\circ} \mathrm{C}, 70 \pm 10 \%$ UR e fotofase de $12 \mathrm{~h}$. As avaliações foram feitas cinco dias após a transferência, anotando a mortalidadeobservada em cada folha, considerando morto todo ácaro que, com o toque de um pincel, apresentava movimento limitado, ou seja, que se locomovia a uma distância inferior ao próprio corpo.

Os dados foram corrigidos pela fórmula de Schneider-Orelli (Alves et al., 2005) e submetidos à análise de variância e as médias submetidas ao teste de Scott - Knott, a nível de 5\%.

\section{RESULTADOS E DISCUSSÃO}

Os dados apresentados evidenciam que houve efeito significativo dos tratamentos sobrea população do T. urticae em todos os experimentos ( $\mathrm{F}=4,675$; $\mathrm{P}<0,05)$ (Tabela 1).

Amaiormortalidadefoiapresentada pelosisolados 1077 B (experimento 2) e 886 F (experimento 4) com $54,9 \%$, seguida pelos isolados $941 \mathrm{D}$ (experimento 3) com $51,0 \%$ e 1024 A (experimento 1) com $45,9 \%$ de mortalidadecorrigida, respectivamente, destacando-os das demais cepas, em seus respectivos experimentos.

Estes dados evidenciam uma ação significativa das cepaseagrandevariabilidadederesultadosencontrados em pesquisas envolvendo seleção e ação tóxica de $B$. thuringiensis. Prova disso é que, utilizando-se produtos formuladosespecíficosparalepidópteros, evidenciou-se diminuiçãona populaçãodeácaros. Contudo,emoutros estudos, houve incremento na população (KRIEG, 1981).

Em insetos, a eficiência, assim como essa variação, é comprovada por diversas pesquisas, como podemos citar resultados em Spodoptera frugiperda para a qual se obteve mortalidade máxima de $45 \%$ (POLANCZYK et al., 2004) a 100\% (Silva-WeRnECK et al., 2000). E, ainda, em teste com 115 isolados na mesma praga, 41 cepas causaram $100 \%$ de mortalidade e 16 entre 75 e $90 \%$ (FATORETTO et al., 2007).

Da mesma forma, para Diatraea saccharalis, em um estudo comparando cinco cepas, apenas uma apresentou $100 \%$ de mortalidade, a segunda melhor cepa matou $69,1 \%$ das lagartas e as outras três cepas apresentaram mortalidade inferior a 3\% (POLANCZYK et al., 2004; Silva-WERNECK et al., 2000; FAtORETTO et al., 2007; GIтAнY et al., 2007). 
Tabela 1 - Mortalidade corrigida $( \pm \mathrm{EP})$ de ácaro rajado Tetranychus urticae por diferentes cepas de Bacillus thuringiensis em quatro experimentos distintos.

\begin{tabular}{|c|c|c|c|}
\hline Cepas & Mortalidade corrigida (\%) & Cepas & Mortalidade corrigida (\%) \\
\hline \multicolumn{4}{|c|}{$\begin{array}{ll}\text { Experimento } 1 \\
\end{array}$} \\
\hline $1024 \mathrm{~A}$ & $45,9 \pm 3,77 \mathrm{~A}$ & PR 13 & $24,0 \pm 2,71 \mathrm{~B}$ \\
\hline 1044 & $34,3 \pm 3,87$ B & $816 \mathrm{~A}$ & $24,0 \pm 2,71 \mathrm{~B}$ \\
\hline 1052 B & $31,7 \pm 1,89 \mathrm{~B}$ & $1009 \mathrm{~K}$ & $22,7 \pm 3,90 \mathrm{C}$ \\
\hline $868 \mathrm{G}$ & $31,7 \pm 3,34 \mathrm{~B}$ & $766 \mathrm{~B}$ & $22,7 \pm 3,37 \mathrm{C}$ \\
\hline R 61 & $30,8 \pm 4,70 \mathrm{~B}$ & R 253 & $20,1 \pm 3,23 \mathrm{C}$ \\
\hline $862 \mathrm{C}$ & $28,0 \pm 9,57 \mathrm{~B}$ & $1075 \mathrm{D}$ & $18,8 \pm 4,10 \mathrm{C}$ \\
\hline $1002 \mathrm{~B}$ & $27,8 \pm 3,90 \mathrm{~B}$ & $1075 \mathrm{~A}$ & $18,8 \pm 3,04 \mathrm{C}$ \\
\hline $775 \mathrm{C}$ & $27,8 \pm 3,90 \mathrm{~B}$ & $862 \mathrm{CF} 2$ & $18,8 \pm 2,34 \mathrm{C}$ \\
\hline $927 \mathrm{R}$ & $27,8 \pm 2,76 \mathrm{~B}$ & PR 7 & $18,8 \pm 4,10 \mathrm{C}$ \\
\hline 872 B & $27,8 \pm 4,36 \mathrm{~B}$ & 1009 SLR & $14,4 \pm 3,91 \mathrm{C}$ \\
\hline SEIVA1 & $26,5 \pm 6,59 \mathrm{~B}$ & $858 \mathrm{EC}$ & $13,6 \pm 1,89 \mathrm{C}$ \\
\hline 1000 Q & $26,5 \pm 3,04 \mathrm{~B}$ & $1010 \mathrm{~J}$ & $13,1 \pm 3,47 \mathrm{C}$ \\
\hline Br 35 & $26,5 \pm 3,04 \mathrm{~B}$ & $1000 \mathrm{~A}$ & $10,2 \pm 3,03 \mathrm{C}$ \\
\hline $76.25 \mathrm{~A}$ & $25,2 \pm 3,77 \mathrm{~B}$ & $979 \mathrm{C}$ & $8,4 \pm 3,48 \mathrm{C}$ \\
\hline R 239 & $24,3 \pm 4,44 \mathrm{~B}$ & 971 & $8,0 \pm 2,36 \mathrm{C}$ \\
\hline \multicolumn{4}{|c|}{ Experimento 2} \\
\hline $1077 \mathrm{~B}$ & $54,9 \pm 6,73 \mathrm{~A}$ & $1075 \mathrm{C}$ & $18,8 \pm 3,04 \mathrm{C}$ \\
\hline $948 \mathrm{G}$ & $33,0 \pm 5,51 \mathrm{~B}$ & 985 & $18,8 \pm 2,34 \mathrm{C}$ \\
\hline CUB.4 & $27,8 \pm 1,95 \mathrm{~B}$ & $937 \mathrm{H} 2$ & $18,8 \pm 1,29 \mathrm{C}$ \\
\hline S 1328 & $25,6 \pm 5,50 \mathrm{~B}$ & 805 B2 & $16,6 \pm 3,33 \mathrm{C}$ \\
\hline E 14 & $25,2 \pm 5,42 \mathrm{~B}$ & SP 16 & $16,6 \pm 3,85 \mathrm{C}$ \\
\hline R 43 & $22,7 \pm 4,36 \mathrm{C}$ & E 15 & $12,7 \pm 3,14 \mathrm{C}$ \\
\hline CST seiva & $22,7 \pm 4,36 \mathrm{C}$ & Br 75 & $12,4 \pm 1,95 \mathrm{C}$ \\
\hline 1043 N-V & $22,2 \pm 7,18 \mathrm{C}$ & SER 3 & $11,8 \pm 3,98 \mathrm{C}$ \\
\hline CST 23.10 & $22,2 \pm 6,63 \mathrm{C}$ & 1000 & $11,5 \pm 3,12 \mathrm{C}$ \\
\hline 1048 NM & $21,4 \pm 3,34 \mathrm{C}$ & MS 2 & $16,6 \pm 4,32 \mathrm{C}$ \\
\hline $775 \mathrm{G}$ & $21,4 \pm 5,49 \mathrm{C}$ & $969 \mathrm{~A}$ & $16,2 \pm 3,04 \mathrm{C}$ \\
\hline 1078 & $21,4 \pm 1,89 \mathrm{C}$ & $858 \mathrm{H}$ & $9,3 \pm 2,64 \mathrm{C}$ \\
\hline $1044 \mathrm{CV}$ & $20,9 \pm 6,60 \mathrm{C}$ & $1077 \mathrm{~A}$ & $8,8 \pm 4,31 \mathrm{C}$ \\
\hline 857 AC2 & $20,1 \pm 3,77 \mathrm{C}$ & $1034 \mathrm{~F}$ & $7,1 \pm 2,57 \mathrm{C}$ \\
\hline 1005 & $20,1 \pm 4,25 \mathrm{C}$ & LMJ 7A & $6,7 \pm 1,93 \mathrm{C}$ \\
\hline \multicolumn{4}{|c|}{ Experimento 3} \\
\hline $941 \mathrm{D}$ & $51,0 \pm 3,23 \mathrm{~A}$ & R 251 & $15,2 \pm 5,87 \mathrm{C}$ \\
\hline R 89 & $27,8 \pm 3,90 \mathrm{~B}$ & $8.7 \mathrm{~L}$ & $14,9 \pm 2,58 \mathrm{C}$ \\
\hline LMJ4A & $20,1 \pm 3,77 \mathrm{C}$ & S 646 & $14,9 \pm 3,23 \mathrm{C}$ \\
\hline $1074 \mathrm{~B}$ & $20,1 \pm 3,77 \mathrm{C}$ & S 165 & $14,9 \pm 3,23 \mathrm{C}$ \\
\hline $1039 \mathrm{C}$ & $20,1 \pm 3,77 \mathrm{C}$ & $27.7 \mathrm{~L}$ & $14,0 \pm 3,64 \mathrm{C}$ \\
\hline T14001 & $18,8 \pm 4,54 \mathrm{C}$ & $1130 \mathrm{~B}$ & $14,0 \pm 3,64 \mathrm{C}$ \\
\hline $1030 \mathrm{~A}$ & $18,8 \pm 3,04 \mathrm{C}$ & $9.7 \mathrm{~L}$ & $14,0 \pm 3,64 \mathrm{C}$ \\
\hline $16.7 \mathrm{~L}$ & $18,8 \pm 3,04 \mathrm{C}$ & $852 \mathrm{~F}$ & $13,1 \pm 4,84 \mathrm{C}$ \\
\hline 36.7 L & $17,9 \pm 4,54 \mathrm{C}$ & $857 \mathrm{CD}$ & $12,7 \pm 3,14 \mathrm{C}$ \\
\hline R 238 & $17,5 \pm 3,90 \mathrm{C}$ & $888 \mathrm{AD}$ & $12,7 \pm 3,14 \mathrm{C}$ \\
\hline SP 13 & $17,5 \pm 3,37 \mathrm{C}$ & S 109 & $11,5 \pm 3,12 \mathrm{C}$ \\
\hline 4D4 & $15,7 \pm 5,07 \mathrm{C}$ & 858 AA & $10,9 \pm 3,75 \mathrm{C}$ \\
\hline $867 \mathrm{BC}$ & $15,3 \pm 3,52 \mathrm{C}$ & RN 1 & $9,8 \pm 1,69 \mathrm{C}$ \\
\hline $1038 \mathrm{~K}$ & $15,3 \pm 3,52 \mathrm{C}$ & 927 A9.15 & $9,7 \pm 3,65 \mathrm{C}$ \\
\hline $933 \mathrm{E}$ & $15,3 \pm 4,02 \mathrm{C}$ & Br 19 & $7,5 \pm 3,63 \mathrm{C}$ \\
\hline \multicolumn{4}{|c|}{ Experimento 4} \\
\hline $886 \mathrm{~F}$ & $54,9 \pm 7,01 \mathrm{~A}$ & $83.26 \mathrm{~A}$ & $15,3 \pm 3,52 \mathrm{C}$ \\
\hline 1001 & $30,4 \pm 3,77 \mathrm{~B}$ & R 5 & $15,3 \pm 4,47 \mathrm{C}$ \\
\hline $862 \mathrm{DE}$ & $27,8 \pm 5,84 \mathrm{~B}$ & 1012 & $14,9 \pm 3,23 \mathrm{C}$ \\
\hline 810 B & $18,3 \pm 5,11 \mathrm{C}$ & $10.7 \mathrm{~L}$ & $14,9 \pm 3,23 \mathrm{C}$ \\
\hline 1010i & $17,9 \pm 3,61 \mathrm{C}$ & $\mathrm{R} 276$ & $14,8 \pm 4,99 \mathrm{C}$ \\
\hline CUB.1 & $17,5 \pm 4,36 \mathrm{C}$ & sps1 (Bti ES) & $14,4 \pm 3,39 \mathrm{C}$ \\
\hline
\end{tabular}


Tabela 1 - Continuação.

\begin{tabular}{llll}
\hline Cepas & Mortalidade corrigida (\%) & Cepas & Mortalidade corrigida (\%) \\
\hline RS 1 & $17,5 \pm 2,76 \mathrm{C}$ & S 244 & $14,4 \pm 3,91 \mathrm{C}$ \\
$1043 \mathrm{~W}$ & $17,5 \pm 3,37 \mathrm{C}$ & $977 \mathrm{FA}$ & $14,4 \pm 4,78 \mathrm{C}$ \\
R 112 & $17,5 \pm 2,76 \mathrm{C}$ & $100.27 \mathrm{~A}$ & $14,4 \pm 4,78 \mathrm{C}$ \\
$34.7 \mathrm{~L}$ & $17,5 \pm 3,37 \mathrm{C}$ & SP 5 & $14,0 \pm 3,64 \mathrm{C}$ \\
$977 \mathrm{~B}$ & $17,5 \pm 3,37 \mathrm{C}$ & 928 & $14,0 \pm 6,60 \mathrm{C}$ \\
Br 42 & $15,7 \pm 5,07 \mathrm{C}$ & Br 34 & $11,5 \pm 2,44 \mathrm{C}$ \\
$1010 \mathrm{~F}$ & $15,7 \pm 3,78 \mathrm{C}$ & $814 \mathrm{~B}$ & $10,6 \pm 3,41 \mathrm{C}$ \\
Br 4 & $15,3 \pm 4,47 \mathrm{C}$ & $2.18 \mathrm{~A}$ & $9,7 \pm 4,14 \mathrm{C}$ \\
$878 \mathrm{~B}$ & $15,3 \pm 4,47 \mathrm{C}$ & R 186 & $9,7 \pm 3,65 \mathrm{C}$ \\
\hline
\end{tabular}

Médias seguidas por mesma letra não diferem entre si ao nível de 5\% pelo teste de Scott - knott.

Especificamente em relação aos ácaros, algumas espécies apresentaram suscetibilidade a $B$. thuringiensis var. israelensis (Glare; O'CALlaghan, 2000). Porém, neste trabalho, as duas cepas dessa espécie resultaram em mortalidade de $18,8 \%$ para a cepa T14001 e de $14,4 \%$ para a cepa sps1 (Bti ES).

Em Cuba, alguns trabalhos avançam em relação à utilização de Bt em ácaros. Nesse sentido, MARQUEZ (1997) caracterizou uma cepa específica para diversas espécies de ácaros daquele país, a LBT-13, com resultados satisfatórios e promissores. Essa caracterização foi importante para o desenvolvimento de outros trabalhos.

Posteriormente, Almaguel (2000) realizou um trabalho de manejo do ácaro T. tumidus em banana, com aplicação da cepa LBT-13, em conjunto com a liberação de um predador e conseguiu reduzir os gastos com o controle dessa praga com acaricidas da ordem de 50 a 70\%. E segundo RojAs (2006), citada por POLANCZYK et al. (2008), a cepa de B. thuringiensis (LBT-13) é eficiente contra três espécies de ácaros (Phyllocoptruta oleivora, Polyphagotarsonemus latus e T. tumidus), causando mortalidade que varia de 70 a $100 \%$, dependendo da espécie em aplicações em cultivos comerciais. Porém, a autora cita esses resultados em aplicações no campo, e não em pesquisa.

Essa possibilidade de ampla variação de resultados pode ser devida a vários fatores inerentes à atividade tóxica de $B$. thuringiensis, tais como: estabilidade da protoxina, solubilização dos cristais, processo proteolítico, ausência e alterações dos receptores específicos nas células epiteliais do intestino médio (Garcia et al., 1982, Fiuza et al., 1996, Peyronnet et al., 1997), evidenciando a sua complexa ação.

A presença de duas ou mais toxinas é comum em estirpes de $B$. thuringiensis e isso pode resultar em interação, negativa ou positiva, provocando efeitos sinérgicos, aditivos ou antagônicos (BENZ, 1971).

Essa interação pode ocorrer também entre proteínas e esporos, como constatado por MIYASONO et al. (1994), que comprovaram o aumento da eficiência de uma suspensão de $B$. thuringiensis com proteínas purificadas após a adição de esporos. A presença de esporos apresenta grande importância na patogenicidade da bactéria, aumentando sua atividade tóxica (Copping; Menn, 2000).

Uma forma eficiente de evitar o efeito da interação entre toxinas diferentes é a purificação, ou seja, isolamento e produção de suspensão contendo apenas a toxina específica ao artrópode alvo, eliminando-se assim a influência de outras toxinas como as VIP's e as exotoxinas (PolanczYK et al., 2004; ARANDA et al.,1996), além de possibilitar a avaliação de sua atividade isolada.

As proteínas Cry5Aa e Cry5Ab, Cry6Aa e Cry6Ba e Cry12Aa são descritas como ativas contra ácaros (Hofte; Whiteley, 1989; Monnerat; Bravo, 2000; CRICKMORE et al., 2008), sendo portanto interessante a tentativa de se formular produtos contendo apenas essas proteínas para realização de experimentos em T. urticae. Para isso, pesquisas futuras envolvendo a caracterização molecular são importantes para se determinar as proteínas envolvidas na atividade tóxica das cepas testadas. Nesse caso, a ação sobre os ácaros pode estar ligada à atuação de uma toxina ainda não caracterizada para a praga, por ação dos esporos ou a atuação em conjunto de toxina e esporos, já que as cepas utilizadas nos experimentos tinham sido previamente caracteri-zadas pelo Laboratório deGenética de Bactérias e Biotecnologia Aplicada da Unesp/Jaboticabal antes de serem utilizadas e não apresentaram as proteínas específicas citadas acima.

Quanto à relação das proteínas Cry com receptores específicos presentes nas microvilosidades das células epiteliais do intestino médio, existe muita controvérsia, pois esta interação engloba inúmeros processos químicos e metabólicos, onde qualquer alteração em um desses processos pode ocasionar uma maior ou menor atividade tóxica. Alguns autores consideram que há uma correlação positiva entre a ligação da toxina no receptor intestinal e a toxicidade (Hofmann et al., 1988; FiuzA et al., 1996). Em contrapartida, outros autores descrevem que o reconhecimento do receptor é necessário, mas não é suficiente para provocar a toxicidade (WolFERSBERGER, 1990). Háainda outros, como ARANDA 
et al. (1996) que afirmaram não existir relação entre a capacidade de ligação aos receptores e a toxicidade, considerando ainda que a atuação da proteína é um processo complexo, no qual a ligação com um receptor é uma etapa necessária, mas insuficiente para conferir atividade inseticida.

Os resultados deste experimento demonstram que, apesar da evidente ação das cepas sobre o ácaro rajado, ainda devem ser realizados mais estudos para se obter níveis mais elevados de mortalidade, bem como elucidar o mecanismo de ação de Bt em ácaros, para, posteriormente, testar produtos formulados mais puros e estáveis às condições ambientais.

\section{CONCLUSÃO}

Bacillus thuringiensis Berliner é patogênico para Tetranychus urticae Koch e os isolados que apresentaram maior mortalidade (1077 B, 886 F, 941 D e 1024 A) são promissores para pesquisas futuras no uso de Bacillus thuringiensis Berliner em T. urticae Koch.

\section{REFERENCIAS}

ALMAGUEL, L. Generalización en Cuba del programa de manejo integrado del ácaro rojo Tetranychus tumidus en plátano. Fitosanidad, v.4, p.93-98, 2000.

ALVES, S.B.; HADDAD, M.L.; MORAES, R.C.B. e REYES, A.E.L. 2005. Utilização de fórmulas de correção de mortalidade. Disponível em: <http:/ / www.lef.esalq. usp.br/cm/intro.php>. Acesso em: 6 out. 2009.

ALVES, S.B.; MORAES, S.A. Quantificação de inoculo de patógenos de insetos. In: ALVES, S.B. (Ed.). Controle microbiano de insetos. Piracicaba: FEALQ, 1998. p.765 778 .

ARANDA, E.; SÁNCHES, J.; PEFEROEN, N.; GUERECA, L.; BRAVO, A. Interaction of Bacillus thuringiensis crystal protein with the midgut epithelial cells of Spodoptera frugiperda (Lepidoptera: Noctuidae). Journal of Invertebrate Pathology, v.68, p.203-212, 1996.

BENZ, G. Synergism of micro-organisms and chemical insecticides. In: BURGES, H.D.; HUSSEY, N.W. (Ed.). Microbial control of insects and mites. Londres: Academic Press, 1971. Chap. 15. p.327-356.

COLLIER, K.F.S.; ALBUQUERQUE, G.S.; LIMA, J.O.G.; PALLINI, A.; MOLINA-RUGAMA, A.J. Neoseiulus idaeus (Acari: Phytoseiidae) as a potential biocontrol agent of the two-spotted spider mite, Tetranychus urticae (Acari: Tetranychidae). In papaya: performance on different prey stage - host plant combinations. Experimental \& Applied Acarology, v.41, p.27-36, 2007.

COPPING, L.G.; MENN, J.J. Review biopesticides: a review of their action, applications and efficacy. Pest Management Science, v.56, p.651-676, 2000.

CRICKMORE, N.; ZEIGLER, D.R.; SCHNEPF, E.; VAN RIE, J.; LERECLUS, D.; BAUM, J.; BRAVO, A.; DEAN, D.H. Bacillus thuringiensis toxin nomenclature. Disponível em: <http://www.lifesci.sussex.ac.uk/home/Neil_ Crickmore/Bt/intro.html>. Acesso em: 26 out. 2008.

FATORETTO, J.C.; SENA, J.A.D.; BARRETO, M.R., LEMOS, M.V.F.; BOIÇA JUNIOR, A.L. Associação de bioensaios e caracterização molecular para seleção de novos isolados de Bacillus thuringiensis efetivos contra Spodoptera frugiperda (J.E.Smith) (Lepidoptera: Noctuidae). Neotropical Entomology, v.36, p.737-745, 2007.

FIUZA, L.M.; LEROUX, N.C.; GOZÉ, E.; FRUTOS, R.; CHARLES, J.F. Binding of Bacillus thuringiensis Cry I toxins to the midgut brush border membrane vesicles of Chilo suppressalis (Lepidoptera: Pyralidae): Evidence of shared binding sites. Applied and Environmental Microbiology, v.62, p.1544-1549, 1996.

GARCIA, M.A.; SIMÕES, M.; HABIB, M.E.M. Possible reasons of resistance in larvae of Spodoptera frugiperda (Abbot \& Smith, 1797) infected by Bacillus thuringiensis var. kurstaki. Revista de Agricultura, v.57, p.215-222, 1982.

GLARE, T.R.; O'CALLAGHAN, M. Bacillus thuringiensis: biology, ecology and safety. Chichester: John Wiley, 2000. 350p.

GITAHY, P.M.; SOUZA, M.T.; MONNERAT, R.G.; ARRIGONI, E.B.; BALDANI, J.I.A Brazilian Bacillus thuringiensis Strain Highly Active to Sugarcane Borer Diatraea saccharalis (Lepidoptera: Crambidae). Brazilian Journal of Microbiology, v.38, p.531-537, 2007.

HANSEN, B.M.; SALAMITOU, S. Virulence of Bacillus thuringiensis. In: CHARLES, J.F.; DELÉCLUSE, A.; NIELSEN-LE ROUX, C. (Ed.). Entomopathogenic bacteria: from laboratory to field application. Netherlands: Kluwer Academic Publishers, 2000. p.41-64.

HOFMANN, C.; VANDERBRUGGEM, H.; HOFTE, H.; VAN RIE, J.; JANSENS, S.; VAN MELLAERT, H Specificity of Bt delta-endotoxins is correlated with the presence of high-affinity binding sites in the brush border membrane of target insect midgets. Proceedings of the National Academy of Sciences of the United States of America, v.85, p.7844-7848, 1988.

HOFTE, H.; WHITELEY, H. R. Insecticidal crystal proteins of Bacillus thuringiensis. Microbiological Reviews, v.53, p.242-255, 1989.

KRIEG, A.; LANGENBRUCH, G.A. Susceptibility of arthropod species to Bacillus thuringiensis. In: BORGES, H.D. (Ed.). Microbial control of pests and plant diseases 1970-1980. New York: Academic Press, 1981. p.837-896. 
MARQUEZ, M.A. Caracterización de un aislado de Bacillus thuringiensis (Berliner) con efecto acaricida. Revista de Protección Vegetal, v.12, p.89-93, 1997.

MIYASONO, M.; INAGAKI, S.; YAMAMOTO, M.; OHBA, K.; ISHIGURO, T.; TAKEDA, R.; HAYASHI, Y. Enhancement of delta-endotoxin activity by toxin-free spore of Bacillus thuringiensis against the diamondback moth, Plutella xylostella. Journal of Invertebrate Pathology, v.63, p.111-112, 1994.

MONNERAT, R.G.; BRAVO, A. Proteínas bioinseticidas produzidas pela bactéria Bacillus thuringiensis: modo de ação e resistência. In: MELO, I.S.; AZEVEDO, J.L. (Ed.). Controle biológico. Jaguariuba: Embrapa Meio Ambiente, 2000. v.3, p.163-200.

MONTEIRO, L.B. Seletividade de inseticidas a Neoseiulus californicus McGregor (Acari: Phytoseiidae) em macieira, no Rio Grande do Sul. Revista Brasileira de Fruticultura, v.23, p.589-592, 2001.

MORAES, G.J.; FLECHTMANN, C.W. Manual de Acarologia: Acarologia Básica e Ácaros de Plantas Cultivadas no Brasil. Ribeirão Preto: Holos Editora, 2008. 308p.

OLIVEIRA, R.C.; NEVES, P.M.O.J.; ALVES, L.F.A. Seleção de fungos entomopatogênicos para controle de Oligonychus yothersi (McGregor) (Acari: Tetranychidae), na cultura da erva-mate (Ilex paraguariensis St. Hill.). Neotropical Entomology, v.33, p.347-351, 2004.

PEYRONNET, O.; VACHON, V.; BROUSSEAU, R. Effect of Bacillus thuringiensis toxins on the membrane potential of lepidopteran insect midgut cells. Applied and Environmental Microbiology, v.63, p.1679-1684, 1997.

POLANCZYK, R.A.; SILVA, R.F.P.; FIUZA, L.M. Isolamento de Bacillus thuringiensis Berliner a partir de amostras de solos e sua patogenicidade para Spodoptera frugiperda (J. E. Smith) (Lepidoptera: Noctuidae). Agro-ciência, v.10, p.209-214, 2004.

POLANCZYK, R.A.; VALICENTE, F.H.; BARRETO, M.R. Utilização de Bacillus thuringiensis no controle de pragas agrícolas na América Latina. In: ALVES, S.B.; LOPES, R.B. (Org.). Controle microbiano de pragas na América Latina: avanços e desfios. Piracicaba: FEALQ, 2008. v.1, p.111-136.

POLETTI, M.; MAIA, A.H.N.; OMOTO, C. Toxicity of neonicotinoid insecticides to Neoseiulus californicus and Phytoseiulus macropilis (Acari: Phytoseiidae) and their impact on functional response to Tetranychus urticae (Acari: Tetranyhchidae). Biological Control, v.40, p.30-36, 2006.
POlETTI, M.; COLlETTE, L.P.; OMOTO, C. Compatibilidade de Agrotóxicos com os Ácaros Predadores Neoseiulus californicus (McGregor) e Phytoseiulus macropilis (Banks) (Acari: Phytoseiidae). BioAssay, v.3, p.3, 2008.

SATO, M.E.; SILVA, M.Z.; CANGANI, K.G.; RAGA, A. Seleções para resistência e suscetibilidade, detecção e monitoramento da resistência de Tetranychus urticae ao acaricida clorfenapir. Bragantia, v.66, p.89-95, 2007.

SILVA, A.L. Controle químico ao ácaro rajado Tetranychus urticae (Koch 1836) Bordeaux \& Dosse 1963, em Tomateiros. Goiânia: Departamento Fitossanitário, Universidade Federal de Goiás, 1971. p.7-15.

SILVA-WERNECK, J.O.; NETO, J.R.M.V.A.; TOSTES, A.N.; FARIA, L.O.; DIAS, J.M.C.S.D. Novo isolado de Bacillus thuringiensis efetivo contra a lagarta-docartucho. Pesquisa agropecuária brasileira, v.35, p.221-227, 2000 .

TAMAI M.A.; ALVES S.B.; NEVES, P.J. Patogenicidade de Beauveria bassiana (Bals.) Vuill. ao ácaro Tetranychus urticae Koch. Scientia Agricola, v.56, p.285-288, 1999.

TAMAI, M.A.; ALVES S.B.; ALMEIDA J.E.M. de; FAION M. Avaliação de fungos entomopatogênicos para o controle de Tetranychus urticae Koch (Acari: Tetranychidae). Arquivos do Instituto Biológico, São Paulo, v.69, n.3, p.77-84, 2002.

WATANABE, M.A.; MORAES, G.J. de; GASTALDO JUNIOR, I.; NICOLELLA, G. Controle biológico do ácaro rajado com ácaros predadores fitoseídeos (Acari: Tetranychidae, Phytoseiidae) em culturas de pepino e morango. Scientia Agrícola, v.51, p.75-81, 1994.

WHITELEY H.R.; SCHNEPF H.E. The molecular biology of parasporal crystal body formation in Bacillus thuringiensis. Annual Review of Microbiology, v.40, p.549576, 1986.

WOLFERSBERGER, M. Specificity and mode of action of Bacillus thuringiensis insecticidal crystal proteins toxic to lepidopteran larvae: recent insights from studies utilizing midgutbrush border membrane vesicles. In: INTERNATIONAL COLLOQUIUM ON IVERTEBRATE PATHOLOHY, 5., 1990, Adelaide. Proceedings. Adelaide: Spciety Invertebrate Pathology, 1990. p.278-282.

Recebido em 27/11/08

Aceito em 14/3/11 